\title{
REVIEW
}

\section{The role of magnesium in the emergency department}

\section{P Kaye, I O'Sullivan}

Emerg Med J 2002;19:288-291

Magnesium has been advocated for the treatment of a variety of conditions seen in emergency medicine. The authors present a systematic review and advice on appropriate indications for its use. Evidence supports its use in severe asthma, eclampsia, and torsade de pointes. There is insufficient evidence to justify its routine use in other emergencies.

M gnesium is the second most abundant intracellular cation and the fourth most abundant cation in the body. It is an essential transmembrane and intracellular modulator of cellular electrical activity. Possible therapeutic uses are broad but until recently, few studies had been undertaken. Numerous clinical trials and other studies are now available in a wide range of conditions. We examine the role of magnesium in the emergency department.

\section{MYOCARDIAL INFARCTION}

There has been considerable interest in magnesium as a treatment to limit myocardial damage in myocardial infarction (MI). Experimentally it has been shown to have a role in myocardial salvage, possibly by inhibiting calcium influx to ischaemic myocytes and/or by reducing coronary tone. It has also been shown to increase the threshold for depolarisation of cardiac myocytes, theoretically reducing the risk of malignant arrhythmia. In healthy humans it can reduce peripheral vascular resistance and increase cardiac output with no effect on cardiac work.

Prior to 1995 a number of small studies and one large study had all produced positive outcomes for magnesium intervention in acute MI. The LIMIT-2 study, a randomised controlled trial (RCT) with 2316 subjects, demonstrated a statistically significant $16 \%$ reduction in all cause mortality for magnesium compared with placebo ( $95 \%$ CI $2 \%$ to $29 \%$ ) mainly because of a reduction in early left ventricular failure. ${ }^{2}$

However, the ISIS-4 study ${ }^{3}$ with 58050 subjects, which assessed the effects on mortality and major morbidity of the addition of intravenous magnesium to standard treatments for acute MI found no benefit. There was no effect on mortality at five weeks and subgroup analysis found no benefit in those patients not receiving thrombolysis or in those presenting within six hours of onset of symptoms. Slightly fewer patients had ventricular fibrillation but more had some other form of cardiac arrest. Overall no difference in risk was seen. In addition, there was a small but significant increase in heart failure, cardiogenic shock and deaths attributed to cardiogenic shock $(1.62 \% \quad v \quad 1.26 \% \quad 2 \mathrm{p}<0.001)$ though the cause of the excess was unclear.

Further small studies subsequent to ISIS-4 have continued to suggest a positive benefit from magnesium in acute MI. ${ }^{45}$ The most recent by Gymlani et $a l^{6}$ demonstrated a significant reduction in deaths from arrhythmia (4\% v 20\%) and from pump failure ( $4 \% v 14 \%)$. The investigators suggested the timing of the treatment was crucial. However, a placebo controlled RCT of intravenous magnesium in 150 patients undergoing primary percutaneous transluminal coronary angioplasty, demonstrated that infusion before, during, or after reperfusion resulted in no improvement in short-term clinical outcome. ${ }^{7}$ At present a further large RCT, the MAGIC $\operatorname{trial}^{8}$ is in progress to specifically address the question of the role of magnesium in the management of acute MI, focusing on early use.

In summary, despite the theoretical benefits magnesium therapy may offer in the management of acute MI there is no good evidence to support its use. The results of the MAGIC trial are awaited with interest.

\section{CARDIAC FAILURE AND MALIGNANT ARRHYTHMIAS}

Hypomagnesaemia is common in left ventricular and biventricular failure, secondary to increased magnesuria mediated by loop diuretics. Positive results in animal experiments led to suggestions that magnesium should form part of the acute treatment protocol for malignant arrhythmias in patients with significant ventricular dysfunction. ${ }^{9-11}$

Bashir et al ${ }^{9}$ performed a randomised, double blind crossover trial of long term oral magnesium supplementation in congestive heart failure. They demonstrated a reduction in non-sustained monomorphic ventricular tachycardia (mVT) of $24 \% \quad(p<0.01)$. The study only contained 21 patients. Similar results were obtained by Sueta et al ${ }^{10}$ in patients with symptomatic heart failure treated acutely with magnesium or placebo. However, only 30 patients were involved, none had a history of symptomatic arrhythmias, and only asymptomatic non-sustained mVT was suppressed. Ceremuzynski et al ${ }^{11}$ demonstrated hypomagnesaemia in 38\% and excessive urinary magnesium in $72 \%$ of 78 patients with congestive heart failure (ejection fraction $<40 \%$ ) on admission. They demonstrated that magnesium treatment reduced episodes of non-sustained mVT

Abbreviations: $\mathrm{MI}$, myocardial infarction; $\mathrm{mVT}$, monomorphic ventricular tachycardia; $\mathrm{AVN}$ atrioventricular nodal; $A F$, atrial fibrillation; $T d P$, torsade de pointes; $E A D$, early after depolarisation; $H F$, hydrofluoric acid 
significantly but again demonstrated no change in the risk of adverse events or death.

The PROMISE study ${ }^{12}$ enrolled 1068 patients with New York Class III/IV heart failure for a RCT of milrinone. All patients had magnesium levels checked during the trial. Serum magnesium was shown not to be an independent risk factor for sudden death or all cause death.

In conclusion there is little evidence to support the routine use of magnesium in patients with ventricular dysfunction presenting with a malignant arrhythmia. Further studies are necessary in view of the biological plausibility of this intervention. Until they are completed it should be reserved for those with proven hypomagnesaemia.

\section{PRIMARY VENTRICULAR TACHYCARDIA}

There is some experimental evidence but no clinical evidence to support the use of magnesium in monomorphic ventricular tachycardia either as treatment or post-event prophylaxis. ${ }^{13}$

\section{CARDIAC ARREST}

Magnesium has been investigated as a first line drug in cardiac arrest in addition to the standard interventions as per Advanced Life Support (ALS) guidelines. No benefit was found in either out of hospital ${ }^{14}$ or in hospital cardiac arrest. ${ }^{15}$

\section{ATRIAL FIBRILLATION}

Magnesium has no effect on sinus node function but does delay atrioventricular nodal (AVN) conduction and prolong the atrial refractory period. Only one small trial on cardioversion of acute atrial fibrillation (AF) has shown any benefit but this included patients with AVN tachycardia and compared the use of magnesium with verapamil rather than placebo. ${ }^{16}$ Another small study ${ }^{17}$ demonstrated some benefit in ventricular rate control though this was no better than digoxin. This was disputed by Frick et al ${ }^{18}$ who demonstrated no effect on heart rate or rate variability in patients with chronic AF.

Magnesium has been shown to act as an indirect antagonist of digoxin at the sarcolemma $\mathrm{Na}+\mathrm{K}+$ ATPase pump and has shown benefit in reducing the incidence of ventricular arrhythmias associated with digoxin toxicity. ${ }^{19}$ The gold standard however remains Fab antibodies.

\section{TORSADE DE POINTES}

Torsade de pointes (TdP) is a form of polymorphic ventricular proarrhythmia associated with QT interval prolongation and the presence of prominent $U$ waves on the resting electrocardiogram (ECG). These ECG changes represent prolonged repolarisation and the development of early after depolarisation (EAD). It may degenerate to ventricular fibrillation.

In myocardial cells repolarisation occurs when the efflux of positive ions (mainly potassium) exceeds the declining influx of sodium and calcium ions. In the long QT syndromes potassium ion channel dysfunction causes an intracellular surplus of positive charge, so delaying ventricular repolarisation. Delay of inactivation of calcium ion channels results in late inflow producing EADs. These may reach threshold amplitude so triggering a ventricular arrhythmia. As the deep subendocardium is most susceptible to prolonged repolarisation and the development of EAD the heterogeneous state of the myocardium results in a specific type of re-entrant arrhythmia. This is reflected in the TdP pattern on ECG.

The long QT syndrome with risk of development of TdP can be congenital but is usually iatrogenic. It is associated with:

- Antiarrhythmics especially class Ia and III

- Phenothiazines and butyrophenones

- Tricyclic antidepressants

- Non-sedative antihistamines
- Some antibiotics especially macrolides

- Antifungals

- Organophosphates

- Cocaine

It is also associated with starvation, bradycardias, and subarachnoid haemorrhage..$^{20} 21$

The aims of treatment in TdP are (a) to shorten the QT interval and (b) to alter the after depolarisation effect. There have been no RCTs of TdP therapy. Present management is based on theoretical concepts supported by experimental evidence and the evidence of small clinical trials and case series. $^{22-24}$

Magnesium, at a dose of $2 \mathrm{~g}(25-50 \mathrm{mg} / \mathrm{kg}$ in children) magnesium sulphate intravenously over one to two minutes, is used to suppress EADs in the emergency situation. In vitro studies have shown it reduces the amplitude of EADs to subthreshold levels by blocking calcium influx. This should be accompanied by correction of hypokalaemia to a serum $\mathrm{K}+$ concentration of $>4.5 \mathrm{mmol} / \mathrm{l}^{25}$ Lignocaine (lidocaine) indirectly suppresses the development of triggered potentials by shortening the action potential duration. However, this effect is inconsistent with a reported success rate of only $50 \%{ }^{26}$

Magnesium with/without potassium infusion should be accompanied by acceleration of the basic heart rate, as there is an inverse relation between rate and the repolarisation duration. $^{21}$

In summary, magnesium is a safe and simple intervention in TdP. It should be the first line drug therapy in TdP followed by attempts to accelerate the heart rate.

\section{BRONCHIAL ASTHMA}

Magnesium acts as a smooth muscle relaxant by altering extracellular calcium influx and intracellular phosphorylation reactions. It may also attenuate the neutrophilic burst associated with inflammatory bronchoconstriction by attenuating mast cell degranulation. The principal trigger for this degranulation is a rise in intracellular calcium, which is antagonised by magnesium. ${ }^{27}$ It has been shown experimentally to augment the bronchodilatory effect of salbutamol and to inhibit histamine induced bronchospasm. ${ }^{28}$

A number of small RCTs have examined intravenous magnesium use in bronchial asthma. Skobeloff et al ${ }^{29}$ reported significant improvements in peak expiratory flow rates and in numbers discharged from the emergency department in adults with severe asthma. This was supported by further studies in adults and children with moderate to severe exacerbations using $2 \mathrm{~g}$ in adults and $20-25 \mathrm{mg} / \mathrm{kg}$ in children. ${ }^{30-32}$ There was no evidence for its efficacy in unselected groups with asthma exacerbation. ${ }^{33}$ One study ${ }^{34}$ however showed no benefit in moderate and severe exacerbations in adults. A Cochrane systematic review in $1999{ }^{35}$ supported the use of intravenous magnesium sulphate in a subgroup of patients with severe acute asthma. Since their meta-analysis three further small RCTs of intravenous magnesium in acute severe childhood asthma have been published two positive and one negative. ${ }^{36-38}$

The possibility of using isotonic magnesium as a vehicle for nebulised $\beta$ agonists has also been investigated with some positive results. ${ }^{39}$

At present, intravenous magnesium seems to be both safe and effective in acute severe asthma in all age groups. A large prospective RCT is necessary to confirm this. Until this is available magnesium should be used as a safe, easy to administer and effective second line agent in acute severe asthma. Nebulised magnesium remains experimental.

\section{ECLAMPSIA}

Eclampsia is the occurrence of one or more epileptiform fits in association with the syndrome of pre-eclampsia. Preeclampsia is a multisystem disorder of pregnancy usually 
associated with hypertension and proteinuria. Eclampsia occurs in one in 2000 deliveries in the developed world and is a factor in $10 \%$ of maternal deaths in the UK.

Magnesium sulphate was first suggested as an anticonvulsant in 1906 and has been widely used in the United States since the 1930s. The suggested mechanism of action is cerebral vasodilatation and/or prevention of ischaemic neuronal damage by blockade of $n$-methyl-d-aspartate (NDMA) receptors. ${ }^{40}$

The Eclampsia Trial Collaborative Group organised a large double blind RCT with two arms comparing magnesium with phenytoin and diazepam. ${ }^{40}$ In the comparison of magnesium and diazepam (910 women) there were significantly fewer recurrent convulsions with magnesium. Treatment with magnesium resulted in 15 fewer women per 100 having further convulsions. There was no significant difference in maternal mortality between the treatments though the trend was favourable for magnesium therapy. There was no effect on the baby's outcome. In the magnesium and phenytoin arm of the study (777 women) there were significantly fewer recurrent convulsions with magnesium. There was also a reduced risk of the need for anaesthesia, an $8 \%$ reduction in ITU admissions, a reduced risk of perinatal death and a reduced risk of neonatal special care for more than seven days. There was a favourable trend for overall maternal outcome with the mortality rate $5.1 \%$ in the diazepam group compared with 3\% in the magnesium group though this failed to reach significant levels.

Two Cochrane Systematic Reviews produced by the Cochrane Pregnancy and Childbirth Group ${ }^{41}{ }^{42}$ support these findings.

Magnesium is the first line drug in eclampsia. It is substantially more effective than both phenytoin and diazepam. The most widely used regimen is a magnesium sulphate $4 \mathrm{~g}$ intravenous bolus followed by $\mathrm{l} \mathrm{g}$ per hour intravenous infusion.

\section{MIGRAINE}

Interest in magnesium as a possible treatment in migraine stems from studies that demonstrate:

(1) Ionised magnesium affects serotonin receptor activity.

(2) An increased ionised calcium/ionised magnesium ratio promotes cerebral vasospasm.

A number of studies have suggested it may have a role in prophylaxis. ${ }^{43}$ Two small studies have also suggested a role in the treatment of acute migraine. Mauskop et al ${ }^{44}$ demonstrated relief of headache within 15 minutes of intravenous magnesium in 32 of 40 patients with migraine, cluster headache, or tension headache. In 18 this was maintained for 24 hours and 16 of these had low serum magnesium levels. Demirkaya et al demonstrated relief of headache in 13 of 15 migraine patients and relief of associated symptoms in all patients compared to partial relief of headache in one patient in the placebo group. ${ }^{45}$

Further large prospective studies are necessary as this intervention in acute migraine is to date unproven though biologically plausible. It is potentially a safe and rapid intervention

\section{ALCOHOL WITHDRAWAL}

Significant magnesium deficiency is a common, though not universal, finding in chronic alcoholism. This is attributable to alcohol induced urinary magnesium loss, malnutrition, gastrointestinal losses, and phosphate and vitamin D deficiency. ${ }^{46}$ A role for magnesium therapy in alcohol withdrawal was suggested by animal studies, which demonstrated that magnesium deficient mice developed ethanol withdrawal features without ethanol exposure. ${ }^{47}$ However, the only RCT of magnesium supplementation in the alcohol withdrawal syndrome in humans found no difference in symptoms or sedation requirement. ${ }^{48} \mathrm{~A}$ recent evidence based practice guideline from the American Society of Addiction Medicine on the pharmacological management of alcohol withdrawal states there is no evidence that magnesium supplementation reduces withdrawal severity or the frequency of delerium or seizures. ${ }^{49}$

\section{Hydrofluoric acid burns}

Hydrofluoric acid (HF) readily penetrates the skin and mucous membranes, causing deep tissue layer destruction. Exposure can produce hypocalcaemia, hypomagnesaemia, hyperkalaemia, cardiac arrhythmias, and death. Fluoride ion chelation is the treatment of choice as free fluoride ions are thought to be responsible for burn progression. Topical and/or parenteral calcium salts are the standard treatments for both dermal and systemic manifestations..$^{50}$ However, magnesium is also an effective fluoride ion chelator. An animal study by Burkhart et al ${ }^{51}$ compared topical preparations of magnesium and calcium in dermal exposure. Histological analysis of the burns demonstrated calcium treatment was associated with less severe and more superficial injury. However, further animal studies comparing intradermal calcium injection with intravenous magnesium suggest that magnesium therapy reduces the severity of HF burns. ${ }^{52}{ }^{53}$ There have been no trials in humans and no comparisons of intravenous magnesium with intravenous or intra-arterial calcium. Magnesium in HF burns remains experimental unless treating proven systemic hypomagnesaemia.

\section{ADVERSE EFFECTS}

Magnesium has minimal side effects in usual therapeutic doses and has a large therapeutic index. At typical doses the main problems are transient facial warmth and flushing though transient hypotension may occur with over-rapid intravenous infusion. At a serum level of $12 \mathrm{mg} / \mathrm{dl}$ abnormal cardiac conduction, absent reflexes and muscle weakness with respiratory depression occur. Regular assessment of the patellar tendon reflex provides a quick and easy method of monitoring for developing toxicity. ${ }^{40}$

\section{CONCLUSION}

Magnesium has a clear role in the emergency management of a number of conditions.

- It should be used as first line therapy in eclampsia and torsade de pointes ventricular tachycardia.

- It has a clearly defined role as a second line therapy in acute severe bronchial asthma.

- Hypomagnesaemia should be considered in patients with biventricular failure presenting with malignant arrhythmias.

- Magnesium should be considered as a temporising measure in cases of severe digoxin toxicity while using Fab antibodies as the specific antidote.

Magnesium is safe and easy to use.

In conclusion, magnesium should be available for immediate use in all emergency departments.

Authors' affiliations

P Kaye, I O'Sullivan, Emergency Department, Bristol Royal Infirmary, Bristol, UK

Conflicts of interest: none.

Funding: none.

\section{REFERENCES}

1 Anon. Magnesium for acute myocardial infarction? Lancet 1991;338:667-8

2 Woods KL, Fletcher S. Longterm outcome after intravenous magnesium sulphate in suspected acute myocardial infarction: the second Leicester Intravenous Magnesium Intervention Trial (LIMIT-2). Lancet 1994:343:816-19. 
3 ISIS-4 Collaborative Group. ISIS-4: A randomised factorial trial assessing early oral captopril, oral mononitrate and intravenous magnesium sulphate in 58050 patients with suspected acute myocardial infarction. Lancet 1995;345:669-85.

4 Raghu C, Peddeswara Rao P, Seshagiri Rao D. Protective effect of intravenous magnesium in acute myocardial infarction following thrombolytic therapy. Int J Cardiol 1999;71:209-15.

5 Parikka H, Toivonen L, Naukkarinen V, et al. Decreases by magnesium of QT dispersion and ventricular arrhythmias in patients with acute myocardial infarction. Eur Heart J 1999:20:1 1 1-20.

6 Gymlani G, Parikh C, Kulkarni AG. Benefits of magnesium in acute MI: timing is crucial. Am Heart J 2000;139:703.

7 Santoro GM, Antoniucci D, Bolognese L, et al. A randomised study of intravenous magnesium in acute myocardial infarction treated with direct coronary angioplasty. Am Heart J 2000;140:891-7.

8 The MAGIC Steering Committee. Rationale and design of the magnesium in coronaries (MAGIC) study. A clinical trial to re-evaluate the efficacy of early administration of magnesium in acute MI. Am Heart $J$ 2000;139:10-14.

9 Bashir Y, Sneddon JF, Staunton HA, et al. Effects of long-term ora magnesium chloride replacement in congestive heart failure secondary to coronary artery disease. Am J Cardiol 1993;72:1156-62.

10 Sueta CA, Clarke SW, Dunlap SH, et al. Effect of acute magnesium administration on the frequency of ventricular arrhythmia in patients with heart failure. Circulation 1994:89:660-6.

11 Ceremuzynski L, Gebalska J, Wolk R, et al. Hypomagnesaemia in heart failure with ventricular arrhythmias. Beneficial effects of magnesium supplementation. J Intern Med 2000; 247:78-86.

12 Eichhom EJ, Tandon PK, DiBianco R, et al. Clinical and prognostic significance of serum magnesium concentration in patients with severe chronic congestive heart failure: the PROMISE Study. J Am Coll Cardiol 1993;21:634-40.

13 Farouque HM, Sanders P, Young GD. Intravenous magnesium sulfate for acute termination of sustained monomorphic ventricular tachycardia associated with coronary artery disease. Am J Cardiol 2000;86:1270-2.

14 Fatovich DM, Prentice DA, Dobb GJ. Magnesium in cardiac arrest. Resuscitation 1997; 35:237-41.

15 Thel MC, Armstrong AL, McNulty SE, et al. Randomised trial of magnesium in in-hospital cardiac arrest. Lancet 1997; 350:1272-6.

16 Gullestad L, Bireland K, Molstad P, et al. The effect of magnesium versus verapamil on supraventricular tachycardia. Clin Cardiol 1993; 16:429-34.

17 Hays JV, Gilman JK, Rubal BJ. Effect of magnesium sulfate on ventricular rate control in atrial fibrillation. Ann Emerg Med 1994;24:61-4.

18 Frick M, Ostergren J, Rosenquist M. Effect of intravenous magnesium on heart rate and heart rate variability in patients with chronic atrial fibrillation. Am J Cardiol 1999;84:104-8

19 Kinlay S, Buckley NA. Magnesium sulfate in the treatment of ventricular arrhythmias due to digoxin toxicity. J Toxicol Clin Toxicol 1995;33:55-9.

20 Viskin S. Long QT syndromes and Torsade de Pointes. Lancet 1999354: 1625-33.

21 Roden DM. A practical approach to torsade de pointes. Clin Cardiol 1997:20:285-90

22 Bailie DS, Inoue H, Kaseda S, et al. Magnesium suppression of early afterdepolarisations and ventricular tachyarrhythmias induced by caesium in dogs. Circulation 1988;77:1395-402.

23 Banai S, Tzivoni D. Drug therapy for torsade de pointes. J Cardiovasc Electrophysiol 1993;4:206-10.

24 Tzivoni D, Banai S, Schuger C, et al. Treatment of Torsades de pointes with magnesium sulphate. Circulation 1988;77:392-7.

25 Advanced Life Support Course Sub-Committee of the Resuscitation Council (UK). Advanced Life Support Course Provider Manual. 3rd edn. London: Resuscitation Council, 1998.

26 Takanaka C, Ogunyankin KO, Sarma JS, et al. Antiarrhythmic and arrhythmogenic actions of varying levels of extracellular magnesium : possible cellular basis for the differences in the efficacy of magnesium and lidocaine in Torsades de Pointes. J Cardiovasc Pharmacol Ther 1997:2:125-34.

27 Monem GF, Kissoon N, Dnicola L. Use of magnesium sulfate in asthma in childhood. Paediatr Ann 1996;25:138-44.
28 Rolla G, Bucca C, Bugiani $M$, et al. Reduction of histamine-induced bronchoconstriction by magnesium in asthmatic subjects. Allergy 1987;42:186-8.

29 Skobeloff EM, Spivey WH, MCNamara RM, et al. Intravenous magnesium sulfate for the treatment of acute asthma in the emergency department. JAMA 1989:262:1210-13.

30 Ciarallo L, Saver AH, Shannon MW. Intravenous magnesium therapy for moderate to severe pediatric asthma : results of a randomised, placebo-controlled trial. J Pediatr 1996;1 29:809-14.

31 Bloch H, Silverman R, Mancherje N, et al. Intravenous magnesium sulfate as an adjunct in the treatment of acute asthma. Chest 1995; 107:1576-81.

32 Devi PR, Kumar L, Singhi SC, et al. Intravenous magnesium sulphate in acute severe asthma not responding to conventional therapy. Indian Paediatr 1997;34:389-97.

33 Green SM, Rothrock SG. Intravenous magnesium for acute asthma: failure to decrease emergency treatment duration or need for hospitalisation. Ann Emerg Med 1992;21:260-5.

34 Tiffany BR, Berk WA, Todd IK, et al. Magnesium bolus or infusion fails to improve expiratory flow in acute asthma exacerbations. Chest 1993; 104:831-4

35 Rowe BH, Bretzlaff JA, Bota GW, et al. Magnesium sulfate treatment for acute asthmatic exacerbations treated in the emergency department. The Cochrane Library lssue 2, 1999.

36 Gurkan F, Haspolat K, Bosnak M, et al. Intravenous magnesium sulphate in the management of moderate to severe acute asthmatic children nonresponding to conventional therapy. Eur J Emerg Med 1999;6:201-5.

37 Scarfone RJ, Loiselle JM, Joffe MD, et al. A randomised trial of magnesium in the emergency department treatment of children with asthma. Ann Emerg Med 2000:36:572-8.

38 Ciarollo L, Brousseau D, Reinert S. Higher dose intravenous magnesium therapy for children with moderate to severe acute asthma. Arch Pediatr Adolesc Med 2000;154:971-83.

39 Nannini LJ Jr, Pendino JC, Corna RA, et al. Magnesium sulfate as a vehicle for nebulised salbutamol in acute asthma. Am J Med 2000:108:193-7.

40 The Eclampsia Trial Collaborative Group. Which anticonvulsant for women with eclampsia? Evidence from the Collaborative Eclampsia Trial. Lancet 1995;345: 1455-63.

41 Duley L, Henderson-Smart DJ. Magnesium sulphate versus phenytoin for eclampsia. The Cochrane Library Issue 3, 2000.

42 Duley L, Henderson-Smart DJ. Magnesium sulphate versus diazepam for eclampsia. The Cochrane Library Issue 3, 2000

43 Peikert A, Wilimzig C, Kohne-Volland R. Prophylaxis of migraine with oral magnesium : results from a prospective, multicentre, placebo-controlled and double blind randomised study. Cephalagia 1996; 16:257-63.

44 Mauskop A, Altura BT, Cracco RQ, et al. Intravenous magnesium sulfate rapidly alleviates headaches of various types. Headache 1996;36:154-60

45 Demirkaya S, Vural O, Dora B, et al. Efficacy of intravenous magnesium sulphate in the treatment of acute migraine attacks. Headache 2001:41:171-7.

46 Ragland G. Electrolyte abnormalities in the alcoholic patient. Emerg Med Clin North Am 1990;8:7.61-73

47 Belknap JK, Berg JH, Coleman RR. Alcohol withdrawal and magnesium deficiency in mice. Pharmacol Biochem Behav 1978;9:1-6.

48 Wilson A, Vulcano B. A double-blind, placebo-controlled trial of magnesium sulfate in the ethanol withdrawal syndrome. Alcohol Clin Exp Res 1984;8:542-5.

49 Mayo-Smith F. Pharmacological management of alcohol withdrawal: a meta-analysis and evidence-based practice guideline. JAMA 1997;278: 144-51.

50 Kirkpatrick J, Enion D, Burd D. Hydrofluoric acid burns:a review. Burns 1995:21:483-93

51 Burkhart K, Brent J, Kirk M, et al. Comparison of topical magnesium and calcium treatment for hydrofluoric acid burns. Ann Emerg Med 1994;24:9-13

52 Williams JM, Hammad A, Cottington EC, et al. Intravenous magnesium in the treatment of hydrofluoric acid burns. Ann Emerg Med 1994:23:464-9.

53 Cox RD, Osgood KA. Evaluation of intravenous magnesium sulfate for the treatment of hydrofluoric acid burns. Clim Toxicol 1994;32:123-36. 